\title{
State Estimation and Tracking Control of Nonlinear Dynamical Systems $^{1}$
}

\author{
S. C. Beeler ${ }^{2}$, H. T. $\operatorname{Tran}^{3}$, and H. T. Banks ${ }^{4}$ \\ Center for Research in Scientific Computation \\ Department of Mathematics \\ North Carolina State University \\ Raleigh, NC 27695
}

\begin{abstract}
In this paper state estimation and feedback tracking control methods for nonlinear systems are presented. The methods, which are based on the "state-dependent Riccati equation", allow the construction of nonlinear estimators and nonlinear feedback tracking controls for a wide class of systems. Our emphasis will be on development of computational methods that are easily implementable as well as efficient. Simulation results of the performance of the nonlinear estimator and tracking control are included. In addition, comparisons with the linear estimator and linear tracking control found through the linearized system are also made.
\end{abstract}

\footnotetext{
${ }^{1}$ This research was supported in part by a DOD/AFOSR MURI Grant AFOSR F49620-95-1-0447 and in part by AFOSR Grant F49620-96-1-0292 (AASERT)

${ }^{2}$ e-mail: scbeeler@eos.ncsu.edu

${ }^{3}$ e-mail: tran@control.math.ncsu.edu

${ }^{4}$ e-mail: htbanks@eos.ncsu.edu
} 


\section{Introduction}

In previous work we performed a comparative survey of several different methods of designing a feedback control for nonlinear systems [1]. Since finding the exact optimal feedback control is, in general, impossible for the nonlinear case, the methods used various approaches to finding an effective suboptimal control. In this paper we will extend one of the nonlinear feedback control methods to treat problems involving tracking control and state estimation, which would allow it to be used in a wide class of real applications.

Both tracking and estimation, like the simple optimal feedback control problem, have been widely studied for linear systems, and methods of formulation of the control and estimator are well known (for example, see $[2,3]$ and the references they contain). In the linear case the tracking problem is solved in two parts: a feedback control determined by the algebraic Riccati equation (ARE), and a time-dependent tracking variable found by solving an ordinary differential equation (incorporating the desired trajectory) backwards from a stable final time. The state estimation problem for linear systems involves the formulation of a state estimator system incorporating the observed measurement of the actual state and a gain matrix found through a second Riccati equation.

To construct observers and tracking controls for the nonlinear case we first hoped to extend the two-term power series control of Garrard [4], given its simplicity and effectiveness in the types of problems studied in [1]. However, since it is based directly on the Hamilton-Jacobi-Bellman equation for optimal feedback control, and the linear tracking control in particular is derived from the Hamiltonian state/costate formulation of the control problem, combining the two proved difficult. Instead, our approaches are based on the state-dependent Riccati equation (SDRE) [5, 6, 7, 8, 9], which is also very simple to calculate, though not quite as effective as the two-term power series method at controlling some chosen test problems in [1]. However, as shown in this paper, the SDRE method is more readily adaptable to the nonlinear tracking and nonlinear state estimation problems, since it is closely related to the ARE-based method used to find those controls in the linear problem.

While there is a large amount of literature available on state estimation for nonlinear systems, a literature search revealed very little material on tracking control for nonlinear systems. The tracking control technique discussed in this paper was developed directly out of the combination of the SDRE for nonlinear control with the linear method for tracking control. The method of state estimation we will describe below is closely related to work by Thau and others $[10,11,12,13]$ on nonlinear estimators which are themselves extensions of the linear state estimation formula.

There are other very different methods for state estimation described in the literature which we decided not to use, mostly because they are difficult to implement, though theoretically solid. A large effort has been made with types of methods that use a nonlinear transformation to change the subject system into a very specific observer form, mostly using many Lie derivatives of the nonlinear functions from the problem measurement and dynamics $[14,15,16,17,18,19,20]$. Other methods use Lie derivatives as well but not in a transformation to a simpler system form [21, 22]. Also, there are methods using a linearization about a family of constant operating points of the system [23], variable-structure system techniques [24], and Lyapunov theory [25]. A number of these methods are compared in the survey paper of Walcott, Corless and Zak [26]. These methods prove difficult to use in many applications, especially problems with complicated nonlinearities in the dynamics or measurement. More specifically, the nonlinear transformation is often very hard to find or may not even exist, higher-order Lie derivatives are often hard to calculate, the linearization around 
the family of solutions may be very difficult, and finding the appropriate Lyapunov function may be quite challenging. There may be restrictions on the types of problems for which various methods can guarantee results theoretically, or even be used at all. The state-dependent Riccati equation based method that we develop involves some approximations and assumptions, but it is straightforward to implement and is applicable to a certain class of nonlinear systems that are important in practice.

In Section 2 of the paper we will give a brief description of the state-dependent Riccati equation in the context of simple feedback control of nonlinear systems. Section 3 will focus on our formulation of the feedback tracking control, and Section 4 on the state estimator, for the nonlinear case. The controls will be implemented on two test problems in Section 5, and the results will be discussed and compared against the linear tracking control and linear and partially nonlinear estimators. Finally, overall conclusions will be given in Section 6 .

\section{The State-Dependent Riccati Equation}

The state-dependent Riccati equation (SDRE) is one method for obtaining a feedback control for nonlinear systems, and is described in detail by Cloutier, D'Souza and Mracek in [9]. It is simply an extension of the constant-valued algebraic Riccati equation used to find the optimal feedback control in the linear quadratic regulator problem, which is well established for finite and infinite dimensional problems (see e.g. [27, 28]). There are also stable and robust algorithms for solving the algebraic Riccati equation available in the literature.

Consider a system of the type

$$
\left\{\begin{array}{l}
\dot{x}(t)=f(x(t))+B u(x(t)) \\
x(0)=x_{0},
\end{array}\right.
$$

which is nonlinear in the state $x \in \Omega \subset R^{m}$ and is linear in the control $u: \Omega \rightarrow R^{k}$, and a quadratic cost functional

$$
J\left(x_{0}, u\right)=\frac{1}{2} \int_{0}^{\infty}\left(x^{T} Q x+u^{T} R u\right) d t
$$

with given constant-valued weighting matrices $Q$ and $R$ of appropriate dimensions. The optimal control problem is to find a state feedback control $u^{*}\left(x^{*}\right)$ which minimizes the cost for all possible initial conditions $x_{0}$.

We begin with the Hamiltonian for this problem, which is defined as

$$
\mathcal{H}(x, u, p)=\frac{1}{2} x^{T} Q x+\frac{1}{2} u^{T} R u+p^{T}(f(x)+B u) .
$$

From the Hamiltonian the necessary conditions for the optimal control are given by

$$
\begin{aligned}
\dot{x}^{*} & =\frac{\partial \mathcal{H}}{\partial p}=f\left(x^{*}\right)+B u^{*} \\
\dot{p}^{*} & =-\frac{\partial \mathcal{H}}{\partial x}=-Q x^{*}-\frac{\partial f^{T}}{\partial x}\left(x^{*}\right) p^{*} \\
0 & =\frac{\partial \mathcal{H}}{\partial u}=R u^{*}+B^{T} p^{*} .
\end{aligned}
$$


From equation (4), the control is given in terms of the costate variable, $p^{*}(t)$, by $u^{*}(t)=-R^{-1} B^{T} p^{*}(t)$.

We will seek a costate of the form $p^{*}(t)=\Pi\left(x^{*}(t)\right) x^{*}(t)$. First, taking the derivative of $p^{*}$ yields $\dot{p}^{*}=\Pi\left(x^{*}\right) \dot{x}^{*}+D_{t} \Pi\left(x^{*}\right) x^{*}$. Then we substitute into this equation the formulas for $\dot{x}^{*}$ and $\dot{p}^{*}$ determined by the necessary conditions (2) and (3), and the formulas for $u^{*}$ and $p^{*}$ given above, resulting in

$$
\Pi\left(x^{*}\right)\left[f\left(x^{*}\right)-B R^{-1} B^{T} \Pi\left(x^{*}\right) x^{*}\right]+D_{t} \Pi\left(x^{*}\right) x^{*}=-Q x^{*}-\frac{\partial f^{T}}{\partial x}\left(x^{*}\right) \Pi\left(x^{*}\right) x^{*},
$$

which can be rewritten as

$$
\Pi\left(x^{*}\right) f\left(x^{*}\right)+\frac{\partial f^{T}}{\partial x}\left(x^{*}\right) \Pi\left(x^{*}\right) x^{*}-\Pi\left(x^{*}\right) B R^{-1} B^{T} \Pi\left(x^{*}\right) x^{*}+Q x^{*}+D_{t} \Pi\left(x^{*}\right) x^{*}=0 .
$$

The term $D_{t} \Pi(x)$ is a somewhat misleading notation; it is the total time derivative of $\Pi(x(t))$ given by

$$
D_{t} \Pi(x)=\sum_{k=1}^{m} \frac{\partial \Pi}{\partial x_{k}}(x) \dot{x}_{k},
$$

and thus has no meaning except when evaluated along a state trajectory $x(t)$ so that $\dot{x}$ can have some value.

For the simpler linear problem, where the dynamics are $f(x)=A_{0} x, x^{*}$ can be factored out of each term in equation (5) so that it becomes

$$
\Pi A_{0}+A_{0}^{T} \Pi-\Pi B R^{-1} B^{T} \Pi+Q=0 .
$$

Equation (6) is now the standard algebraic Riccati equation with a constant-valued solution matrix $\Pi$. The resulting optimal feedback control is given by $u^{*}\left(x^{*}\right)=-R^{-1} B^{T} \Pi x^{*}$.

The SDRE method involves mimicking the above use of the Riccati equation by rewriting the nonlinear function of $x$ in (1) as $f(x)=A(x) x$. Note that the choice of the state-dependent matrix function $A$ is not unique, and different controls will result from different choices of $A$. With $f$ rewritten in this way equation (5) becomes

$$
\begin{aligned}
& \Pi\left(x^{*}\right) A\left(x^{*}\right) x^{*}+A^{T}\left(x^{*}\right) \Pi\left(x^{*}\right) x^{*}+\sum_{i=1}^{m} x_{i}^{*}\left(\frac{\partial A_{1 \rightarrow m, i}}{\partial x}\left(x^{*}\right)\right)^{T} \Pi\left(x^{*}\right) x^{*} \\
&-\Pi\left(x^{*}\right) B R^{-1} B^{T} \Pi\left(x^{*}\right) x^{*}+Q x^{*}+D_{t} \Pi\left(x^{*}\right) x^{*}=0,
\end{aligned}
$$

which can then be rewritten as

$$
\begin{aligned}
& {\left[\Pi\left(x^{*}\right) A\left(x^{*}\right)+A^{T}\left(x^{*}\right) \Pi\left(x^{*}\right)-\Pi\left(x^{*}\right) B R^{-1} B^{T} \Pi\left(x^{*}\right)+Q\right.} \\
&\left.+\sum_{i=1}^{m} x_{i}^{*}\left(\frac{\partial A_{1 \rightarrow m, i}}{\partial x}\left(x^{*}\right)\right)^{T} \Pi\left(x^{*}\right)+D_{t} \Pi\left(x^{*}\right)\right] x^{*}=0,
\end{aligned}
$$

where the $A$-column derivatives are given by 


$$
\frac{\partial A_{1 \rightarrow m, i}}{\partial x}=\left(\begin{array}{ccc}
\partial A_{1 i} / \partial x_{1} & \cdots & \partial A_{1 i} / \partial x_{m} \\
\vdots & \ddots & \vdots \\
\partial A_{m i} / \partial x_{1} & \cdots & \partial A_{m i} / \partial x_{m}
\end{array}\right)
$$

This equation can be divided into the state-dependent Riccati equation

$$
\Pi(x) A(x)+A^{T}(x) \Pi(x)-\Pi(x) B R^{-1} B^{T} \Pi(x)+Q=0,
$$

and two extra terms

$$
\sum_{i=1}^{m} x_{i}\left(\frac{\partial A_{1 \rightarrow m, i}}{\partial x}(x)\right)^{T} \Pi(x)+D_{t} \Pi(x)=0 .
$$

In the SDRE method one assumes the extra terms remain small and ignores them (thus, among other things, removing the necessity of specifying a trajectory $x(t)$ so that $D_{t} \Pi(x)$ can be evaluated in the equation). This assumption creates a suboptimal feedback control based on the solution to the Riccati equation (8) and given by

$$
u(x)=-R^{-1} B^{T} \Pi(x) x .
$$

In almost all problems the SDRE solution $\Pi(x)$ of $(8)$ is much more difficult to find than for the constant-valued case (6). Wernli and Cook [7] propose a method for solving the Riccati equation (8) using a power series expansion, which we will follow. We begin by splitting $A$ into a constant part and a state-dependent part as $A(x)=A_{0}+\varepsilon \Delta A(x)$, where $\varepsilon$ is a temporary variable used for the expansion that will be set to 1 later. We next write $\Pi$ as a power series in $\varepsilon$, as

$$
\Pi(x, \varepsilon)=\sum_{n=0}^{\infty} \varepsilon^{n} L_{n}(x),
$$

where $\Pi$ as well as each $L_{n}$ is symmetric. Substituting these expansions into the state-dependent Riccati equation (8), we find

$$
\begin{aligned}
&\left(\sum_{n=0}^{\infty} \varepsilon^{n} L_{n}(x)\right)\left(A_{0}\right.+\varepsilon \Delta A(x))+\left(A_{0}^{T}+\varepsilon \Delta A^{T}(x)\right)\left(\sum_{n=0}^{\infty} \varepsilon^{n} L_{n}(x)\right) \\
&-\left(\sum_{n=0}^{\infty} \varepsilon^{n} L_{n}(x)\right) B R^{-1} B^{T}\left(\sum_{n=0}^{\infty} \varepsilon^{n} L_{n}(x)\right)+Q=0 .
\end{aligned}
$$

We then match terms with the same powers of $\varepsilon$ to obtain the following set of equations to determine $L_{n}$ :

$$
\begin{aligned}
L_{0} A_{0}+A_{0}^{T} L_{0}-L_{0} B R^{-1} B^{T} L_{0}+Q & =0 \\
L_{1}\left(A_{0}-B R^{-1} B^{T} L_{0}\right)+\left(A_{0}^{T}-L_{0} B R^{-1} B^{T}\right) L_{1}+L_{0} \Delta A+\Delta A^{T} L_{0} & =0 \\
L_{n}\left(A_{0}-B R^{-1} B^{T} L_{0}\right)+\left(A_{0}^{T}-L_{0} B R^{-1} B^{T}\right) L_{n}+L_{n-1} \Delta A+\Delta A^{T} L_{n-1} & \\
-\sum_{k=1}^{n-1}\left(L_{k} B R^{-1} B^{T} L_{n-k}\right) & =0 .
\end{aligned}
$$


Equation (12) is the standard Riccati equation for the linear part of the system, $A_{0}$, and is easily solved, but equations (13) and (14) may be tedious to solve if the function $\Delta A(x)$ is complicated. However, for a certain class of nonlinear functions, the higher-order terms in the SDRE expansion are much easier to consider. Specifically, we consider those problems in which $\Delta A$ has the same function of $x$ in all of its entries, so it can be written as $\Delta A(x)=g(x) \Delta A_{C}$ with a constant-valued matrix $\Delta A_{C}$ (both of the examples in Section 5 are of this type). By defining $L_{n}(x)=g^{n}(x)\left(L_{n}\right)_{C}$, where $\left(L_{n}\right)_{C}$ is a constant-valued matrix, we can factor out $g^{n}(x)$ from (14) to obtain

$$
\begin{aligned}
\left(L_{n}\right)_{C}\left(A_{0}-B R^{-1} B^{T} L_{0}\right)+\left(A_{0}^{T}-L_{0} B R^{-1} B^{T}\right)\left(L_{n}\right)_{C} & +\left(L_{n-1}\right)_{C} \Delta A_{C}+\Delta A_{C}^{T}\left(L_{n-1}\right)_{C} \\
& -\sum_{k=1}^{n-1}\left(\left(L_{k}\right)_{C} B R^{-1} B^{T}\left(L_{n-k}\right)_{C}\right)=0 .
\end{aligned}
$$

The above equation is a constant-valued matrix Lyapunov equation, for which stable and efficient algorithms exist in the literature, so this class of SDRE problems can be solved to as many terms of the power series as desired. Once as many $L_{n}$ terms as desired have been found, the control is obtained by substituting them back into (11) and (10) with $\varepsilon$ set to 1 .

Some theoretical results are available for the state-dependent Riccati equation and the suboptimal feedback control law derived from it. Wernli and Cook [7] show the existence and asymptotic stability of the controlled system, given certain assumptions on the properties of system. Cloutier, D'Souza and Mracek [9] prove, given certain other assumptions, properties of local and global stability, robustness and suboptimality for the control. Hammett, Hall and Ridgely [29] discuss the issues of controllability and stabilizability (properties important to the proofs in [9]) in regards to the SDRE method of controlling nonlinear systems.

The SDRE method can be calculated very quickly for the case described above with $\Delta A(x)=$ $g(x) \Delta A_{C}$, but this assumption on the form of $\Delta A$ does limit the problems for which the SDRE approach is most useful. There is also the drawback that this method of control ignores the extra terms in equation (9) in setting up the SDRE, and additionally the power series solution is only an approximation to the exact solution of the SDRE (in particular it is less accurate farther from the power series expansion point of the origin). These approximations lead to only a suboptimal feedback control, although the application of the method to test problems in [1] shows generally very good results.

\section{Tracking Control for Nonlinear Systems}

In this section we will derive an analogous SDRE-based solution to the feedback tracking control

problem for a nonlinear system in a manner similar to the derivation in [3] for a linear system. We focus on the differential equation system

$$
\left\{\begin{array}{l}
\dot{x}(t)=f(x(t))+B u(x(t), t) \\
x(0)=x_{0} \\
y(t)=H x(t)
\end{array}\right.
$$

This is the same as in the previous section with the addition of the variable $y$, which is the signal we want to follow along a desired trajectory. We take $y$ as a linear function of the state variables for now. The cost function for the tracking problem, with a desired trajectory $r(t)$, is given by 


$$
J\left(x_{0}, u\right)=\frac{1}{2} \int_{0}^{\infty}\left((y-r)^{T} Q(y-r)+u^{T} R u\right) d t .
$$

To prepare for the application of the SDRE, we rewrite the nonlinear function as $f(x)=A(x) x$. With this notation, the Hamiltonian is given by

$$
\mathcal{H}(x, u, p)=\frac{1}{2}(H x-r)^{T} Q(H x-r)+\frac{1}{2} u^{T} R u+p^{T}(A(x) x+B u) .
$$

The necessary conditions for the optimal control are

$$
\begin{aligned}
\dot{x}^{*} & =\frac{\partial \mathcal{H}}{\partial p}=A\left(x^{*}\right) x^{*}+B u^{*} \\
\dot{p}^{*} & =-\frac{\partial \mathcal{H}}{\partial x}=-H^{T} Q\left(H x^{*}-r\right)-A^{T}\left(x^{*}\right) p^{*}-\sum_{i=1}^{m} x_{i}^{*}\left(\frac{\partial A_{1 \rightarrow m, i}}{\partial x}\left(x^{*}\right)\right)^{T} p^{*} \\
0 & =\frac{\partial \mathcal{H}}{\partial u}=R u^{*}+B^{T} p^{*},
\end{aligned}
$$

with the $A$-column derivatives as defined in equation (7). From equation (17), the control is given by the form $u^{*}(t)=-R^{-1} B^{T} p^{*}(t)$.

We seek a costate of the form $p^{*}(t)=\Pi\left(x^{*}(t)\right) x^{*}(t)+s(t)$ (with a time-dependent tracking variable $s$ added to the version from Section 2) which satisfies the necessary conditions. As before, we take the derivative of $p^{*}$ to obtain $\dot{p}^{*}=\Pi\left(x^{*}\right) \dot{x}^{*}+\dot{s}+D_{t} \Pi\left(x^{*}\right) x^{*}$. Then we substitute in the formulas for $\dot{x}^{*}$ and $\dot{p}^{*}$ from the necessary conditions (15) and (16), and the above formulas for $u^{*}$ and $p^{*}$, yielding

$$
\begin{gathered}
\Pi\left(x^{*}\right) \quad\left[A\left(x^{*}\right) x^{*}-B R^{-1} B^{T}\left(\Pi\left(x^{*}\right) x^{*}+s\right)\right]+\dot{s}+D_{t} \Pi\left(x^{*}\right) x^{*}=-H^{T} Q\left(H x^{*}-r\right) \\
-A^{T}\left(x^{*}\right)\left(\Pi\left(x^{*}\right) x^{*}+s\right)-\sum_{i=1}^{m} x_{i}^{*}\left(\frac{\partial A_{1 \rightarrow m, i}}{\partial x}\left(x^{*}\right)\right)^{T}\left(\Pi\left(x^{*}\right) x^{*}+s\right),
\end{gathered}
$$

which can be rewritten as

$$
\begin{aligned}
& {\left[\Pi\left(x^{*}\right) A\left(x^{*}\right)+A^{T}\left(x^{*}\right) \Pi\left(x^{*}\right)-\Pi\left(x^{*}\right) B R^{-1} B^{T} \Pi\left(x^{*}\right)+H^{T} Q H\right] x^{*}+\left[\dot{s}+A^{T}\left(x^{*}\right) s\right.} \\
& \left.-\Pi\left(x^{*}\right) B R^{-1} B^{T} s-H^{T} Q r+\sum_{i=1}^{m} x_{i}^{*}\left(\frac{\partial A_{1 \rightarrow m, i}}{\partial x}\left(x^{*}\right)\right)^{T}\left(\Pi\left(x^{*}\right) x^{*}+s\right)+D_{t} \Pi\left(x^{*}\right) x^{*}\right]=0 .
\end{aligned}
$$

This separates the equation into two parts: a state-dependent Riccati equation for determining $\Pi(x)$ with which to find the feedback control gain, and an ODE for determining the tracking variable $s(t)$.

In the non-tracking feedback control problem in Section 2, the terms involving derivatives of $A$ and $\Pi$ in (9) were assumed to be small and were neglected, thus reducing the problem down to Riccati equation form. This neglecting of terms is what makes the SDRE method only a suboptimal means of control. However, now there is a second part to the problem, the tracking variable equation given by 


$$
\dot{s}+A^{T}(x) s-\Pi(x) B R^{-1} B^{T} s-H^{T} Q r+\sum_{i=1}^{m} x_{i}\left(\frac{\partial A_{1 \rightarrow m, i}}{\partial x}(x)\right)^{T}(\Pi(x) x+s)+D_{t} \Pi(x) x=0 .
$$

We note that the solution to this equation is state-dependent (i.e., $s$ should actually be written as $s(t, x))$ through the presence of $A(x)$ and $\Pi(x)$ even without the derivative terms, so by keeping the derivative terms in the equation we can include their effects in the control design without drastically changing the nature of the problem.

Solving the tracking equation is more difficult for this nonlinear problem than for the linear case described in [3]. In the linear problem the ODE is solved, offline, backwards from an assumed final time value $s\left(T_{f}\right)=0$. For the nonlinear case, $s(t, x)$ must still be computed offline (since the value of $s$ at $t=0$ is unknown), but now this computation must include the dependence of the equation on $x$ through the now state-dependent $A$ and $\Pi$ as well as the derivative terms not present in the linear case. We do this by solving for $s$ using a trajectory $x_{n o m}(t)$ found with the state equation $\dot{x}_{n o m}=A\left(x_{n o m}\right) x_{n o m}-B R^{-1} B^{T}\left(\Pi\left(x_{n o m}\right) x_{n o m}+s\right)$, coupled to equation (18). Given the initial condition $x_{n o m}(0)=x_{0}$ and the final condition $s\left(T_{f}, x_{n o m}\left(T_{f}\right)\right)=0$, this results in a twopoint boundary value problem which can be discretized and solved with a finite difference method for $x_{n o m}(t)$ and $s\left(t, x_{n o m}(t)\right)$. Note that the coupling of the $s$ and $x_{n o m}$ equations provides the trajectory $x_{n o m}(t)$ necessary to evaluate the $D_{t} \Pi\left(x_{n o m}\right)$ term in (18).

We use the notation $x_{n o m}$ to clarify that this is a nominal trajectory solved offline, before actual implementation of the feedback control on the system. If the system dynamics are known precisely then it will match the actual trajectory precisely. However, for example, in Section 5 the control is applied to a problem with random noise in the $\dot{x}$ system but not in the nominal $\dot{x}_{n o m}$ system, to represent a problem where an actual physical system $\dot{x}$ has some unpredictable noise which the model $\dot{x}_{n o m}$ is unable to include. In the offline calculation of $s$ coupled with $x_{n o m}$ in the TPBV problem, differences between $x$ and $x_{n o m}$ due to an altered initial value or some variation or noise will result in an inaccurate $s$ since it is found using an inaccurate $x_{n o m}$. We tacitly assume that $x_{n o m}$ is a good prediction of the actual state behavior, and that any small differences will not drastically impact the effectiveness of the control. Another area of concern in this tracking control method is the fact, discussed in Section 2, that since we are finding a power series based solution to the SDRE, it will be more inaccurate the farther the state is from the expansion point, which is the origin. This is particularly important for the tracking problem, since we specifically want the state to follow a certain nonzero trajectory.

To summarize, the control formula

$$
u(x, t)=-R^{-1} B^{T}(\Pi(x) x+s(t, x))
$$

is found by, first, solving the SDRE

$$
\Pi(x) A(x)+A^{T}(x) \Pi(x)-\Pi(x) B R^{-1} B^{T} \Pi(x)+H^{T} Q H=0
$$

for $\Pi(x)$ in the manner described in Section 2. The second part of the control design process is then to find the tracking variable $s\left(t, x_{n o m}\right)$ from

$$
\left\{\begin{aligned}
\dot{s}= & -A^{T}\left(x_{n o m}\right) s+\Pi\left(x_{n o m}\right) B R^{-1} B^{T} s+H^{T} Q r \\
& -\sum_{i=1}^{m}\left(x_{n o m}\right)_{i}\left(\frac{\partial A_{1 \rightarrow m, i}}{\partial x_{n o m}}\left(x_{n o m}\right)\right)^{T}\left(\Pi\left(x_{n o m}\right) x_{n o m}+s\right)-D_{t} \Pi\left(x_{n o m}\right) x_{n o m} \\
\dot{x}_{n o m}= & A\left(x_{n o m}\right) x_{n o m}-B R^{-1} B^{T}\left(\Pi\left(x_{n o m}\right) x_{n o m}+s\right)
\end{aligned}\right.
$$


in a two-point boundary value problem with $x_{n o m}(0)=x_{0}$ and $s\left(T_{f}, x_{n o m}\left(T_{f}\right)\right)=0$.

For a nonlinear tracking signal $y(t)=H(x(t)) x(t)$, the control problem becomes somewhat more complicated. The state-dependence of $H$ affects the control formulation in a similar way to that of $A$, resulting in the Riccati and tracking variable equations in (20) and (18) being expanded into the following forms:

$$
\begin{gathered}
\Pi(x) A(x)+A^{T}(x) \Pi(x)-\Pi(x) B R^{-1} B^{T} \Pi(x)+H^{T}(x) Q H(x)=0 \\
\dot{s}+A^{T}(x) s-\Pi(x) B R^{-1} B^{T} s- \\
H^{T}(x) Q r+\sum_{i=1}^{m} x_{i}\left(\frac{\partial H_{1 \rightarrow n, i}}{\partial x}(x)\right)^{T} Q(H(x) x-r) \\
+\sum_{i=1}^{m} x_{i}\left(\frac{\partial A_{1 \rightarrow m, i}}{\partial x}(x)\right)^{T}(\Pi(x) x+s)+D_{t} \Pi(x) x=0 .
\end{gathered}
$$

The tracking variable equation is only slightly changed, adding one more term and making $H$ statedependent in another, neither of which strongly affects the solution process. The SDRE contains the added state-dependence of $H$ in the $H^{T} Q H$ term, which can have more important consequences. If $H(x)$ is not a very complicated formula as was discussed for $A(x)$ in Section 2, such as $H(x)=$ $H_{0}+g(x) \Delta H_{C}$, then it may simply add terms to the equivalent of the higher-order equations (13) and (14) in the power series solution of equation (22). A problem arises if $H(x)$ has no constantvalued part $H_{0}$, but only higher-order parts. This will cause the $Q$-based term to vanish from the tracking problem equivalent of the first equation in the power series solution (12), which results in only a trivial solution to that equation and causes difficulties in the higher-order equations as well. This makes finding a usable feedback control with this method very difficult for that case. However, the linear tracking signal is sufficient for a large number of tracking problems, and we will consider only that form of the problem for now.

\section{State Estimation for Nonlinear Systems}

The method we chose for the state estimation is related to the linear-system case $[2,3]$ and to previous work on nonlinear systems in the literature $[10,11,12,13]$. We consider a system of ODEs with nonlinear dynamics and a nonlinear measurement of the form

$$
\left\{\begin{array}{l}
\dot{x}(t)=f(x(t))+B u\left(x_{e}(t), t\right) \\
z(t)=c(x(t)) .
\end{array}\right.
$$

The control for a tracking problem is given by $u\left(x_{e}, t\right)=-R^{-1} B^{T}\left(\Pi\left(x_{e}\right) x_{e}+s\left(t, x_{n o m}\right)\right)$ as discussed in the last section except now in terms of the estimated state $x_{e}$.

We look for a state estimator of type $\dot{x}_{e}=f_{c}\left(x_{e}, t\right)+F\left(z, x_{e}\right)$. If the error in the estimation is $e=x-x_{e}$, then

$$
\dot{e}=\dot{x}-\dot{x}_{e}=f(x)+B u\left(x_{e}, t\right)-f_{c}\left(x_{e}, t\right)-F\left(c(x), x_{e}\right) .
$$

Let $f_{c}\left(x_{e}, t\right)=f\left(x_{e}\right)+B u\left(x_{e}, t\right)-F\left(c\left(x_{e}\right), x_{e}\right)$. This leads to

$$
\dot{e}=\left(f(x)-F\left(c(x), x_{e}\right)\right)-\left(f\left(x_{e}\right)-F\left(c\left(x_{e}\right), x_{e}\right)\right) .
$$


As an indication of a good estimator, we want the error to be asymptotically stable, so that $e(t) \rightarrow 0$ (or $x_{e}(t) \rightarrow x(t)$ ) as $t \rightarrow \infty$. We want to choose a function $F$ which will satisfy this condition.

For a linear problem, satisfying the stability condition is fairly straightforward, as described in $[2,3]$. The dynamics in this case become $f(x)=A_{0} x$, the measurement becomes $c(x)=C_{0} x$ and we write the unknown function $F\left(z, x_{e}\right)=L_{0} z=L_{0} C_{0} x$, so that we are seeking a constant-valued gain matrix $L_{0}$. The problem then reduces to $\dot{e}=\left(A_{0}-L_{0} C_{0}\right) e$. This is asymptotically stable when all the eigenvalues of $A_{0}-L_{0} C_{0}$ have negative real parts, so an $L_{0}$ must be found which results in such eigenvalues. Such an $L_{0}$ is guaranteed to exist if the pair $\left(A_{0}, C_{0}\right)$ is observable.

An "optimal" choice of $L_{0}$ can be made by considering this problem as an optimal feedback control problem. Since the eigenvalues of a matrix are the same as those of its transpose, we can change the problem into forcing the eigenvalues of $\left(A_{0}-L_{0} C_{0}\right)^{T}=A_{0}^{T}-C_{0}^{T} L_{0}^{T}$ to have negative real parts. However, we note that this form of the system is related to the feedback control problem with $\tilde{A}=A_{0}^{T}, \tilde{B}=C_{0}^{T}$ and $\tilde{L}=L_{0}^{T}$ :

$$
\begin{aligned}
\dot{\tilde{x}} & =\tilde{A} \tilde{x}+\tilde{B} \tilde{u} \\
\tilde{J} & =\frac{1}{2} \int_{0}^{\infty}\left(\tilde{x}^{T} M \tilde{x}+\tilde{u}^{T} N \tilde{u}\right) d t
\end{aligned}
$$

with a control $\tilde{u}=-\tilde{L} \tilde{x}$. The optimal feedback gain for this problem, which best stabilizes the system given the cost functional, is given by $\tilde{L}=N^{-1} \tilde{B}^{T} \Sigma$, with $\Sigma$ solving the Riccati equation

$$
\Sigma \tilde{A}+\tilde{A}^{T} \Sigma-\Sigma \tilde{B} N^{-1} \tilde{B}^{T} \Sigma+M=0,
$$

or, rewritten in terms of the original variables, $L_{0}=\Sigma C_{0}^{T} N^{-1}$ and

$$
\Sigma A_{0}^{T}+A_{0} \Sigma-\Sigma C_{0}^{T} N^{-1} C_{0} \Sigma+M=0 .
$$

This yields the "optimal" state estimation gain matrix $L_{0}$ for constructing the state estimator

$$
\dot{x}_{e}=A_{0} x_{e}+B u\left(x_{e}, t\right)+L_{0}\left(z-C_{0} x_{e}\right) .
$$

Several papers in the literature have expanded on this state estimation formula to include nonlinear problems. Thau [10] considers systems with the linear and nonlinear parts split, such as

$$
\dot{x}=A_{0} x+g(x)+B u,
$$

where $g(x)$ contains only second-order and higher terms, and there is a linear measurement function $z=C_{0} x$. Thau's estimator is of the form

$$
\dot{x}_{e}=A_{0} x_{e}+g\left(x_{e}\right)+B u+L_{0}\left(z-C_{0} x_{e}\right),
$$

with the gain matrix $L_{0}$ calculated so that the eigenvalues of the linear part of the problem, $A_{0}-L_{0} C_{0}$, have negative real parts. This can be done with a Riccati equation in the manner described earlier. This leads to the following error equation:

$$
\dot{e}=\left(A_{0}-L_{0} C_{0}\right) e+g(x)-g\left(x_{e}\right) .
$$

Thus if $g(x)$ is locally Lipschitz, the estimator will locally converge asymptotically. 
Kou, Elliott and Tarn [11] consider a problem with nonlinear dynamics and measurement function and present a condition on the constant gain matrix $L_{0}$ which guarantees that the estimator

$$
\dot{x}_{e}=f\left(x_{e}\right)+B u+L_{0}\left(z-c\left(x_{e}\right)\right)
$$

is asymptotically stable and the error decreases exponentially. However, it is often very difficult or impossible to find $L_{0}$ which satisfies this, and so this is often not very practical. Mielczarski [12] and $\mathrm{Hu}$ [13] use estimators of the type in equation (26), finding $L_{0}$ by separating the dynamics (as well as the measurement) into linear and nonlinear parts like Thau did in equation (24), and using those linear parts to find the gain matrix. They do this separation by linearizing $f(x)$ and $c(x)$ about the origin or some other expansion point to obtain matrices $A_{0}$ and $C_{0}$ for the linear parts, then finding $L_{0}$ which results in all eigenvalues of $A_{0}-L_{0} C_{0}$ having negative real parts.

The method we will describe here extends this nonlinear estimator technique further in a somewhat different direction. Instead of using a completely linearized system for finding the gain matrix $L_{0}$, we will use a state-dependent Riccati equation to solve for the gain from the nonlinear system itself. Rewriting $f$ and $c$ into matrix multiplication form as $f(x)=A(x) x$ and $c(x)=C(x) x$, and replacing $F$ by $F\left(c(x), x_{e}\right)=L\left(x_{e}\right) C(x) x$, we find that equation (23) becomes

$$
\dot{e}=\left(A(x)-L\left(x_{e}\right) C(x)\right) x-\left(A\left(x_{e}\right)-L\left(x_{e}\right) C\left(x_{e}\right)\right) x_{e} .
$$

We further manipulate the equation by adding and subtracting terms to change it into a form more like equation (25):

$$
\begin{aligned}
\dot{e} & =\left(A(x)-L\left(x_{e}\right) C(x)\right) x-\left(A\left(x_{e}\right)-L\left(x_{e}\right) C\left(x_{e}\right)\right) x_{e}+(1-1)\left(A\left(x_{e}\right)-L\left(x_{e}\right) C\left(x_{e}\right)\right) x \\
& =\left(A\left(x_{e}\right)-L\left(x_{e}\right) C\left(x_{e}\right)\right) e+\left(A(x)-A\left(x_{e}\right)-L\left(x_{e}\right) C(x)+L\left(x_{e}\right) C\left(x_{e}\right)\right) x
\end{aligned}
$$

We seek a gain $L$ such that $\dot{e}=\left(A\left(x_{e}\right)-L\left(x_{e}\right) C\left(x_{e}\right)\right) e$ is asymptotically stable, and assume that the remaining term is small (which is reasonable if $x$ is small, or $x$ and $x_{e}$ are close and $A$ and $C$ satisfy certain regularity properties). In analogy to the linear case, the "optimal" state-dependent estimator gain will be computed from a state-dependent Riccati equation as outlined below.

As in the linear case, we consider the transpose of the system, $A^{T}(x)-C^{T}(x) L^{T}(x)$. From there we set up the related feedback control problem with $\tilde{A}=A^{T}, \tilde{B}=C^{T}$ and $\tilde{L}=L^{T}$ :

$$
\begin{aligned}
\dot{\tilde{x}} & =\tilde{A}(\tilde{x}) \tilde{x}+\tilde{B}(\tilde{x}) \tilde{u} \\
\tilde{J} & =\frac{1}{2} \int_{0}^{\infty}\left(\tilde{x}^{T} M \tilde{x}+\tilde{u}^{T} N \tilde{u}\right) d t \\
\tilde{u} & =-\tilde{L}(\tilde{x}) \tilde{x} .
\end{aligned}
$$

The optimal feedback gain is now given by $\tilde{L}(\tilde{x})=N^{-1} \tilde{B}^{T}(\tilde{x}) \Sigma(\tilde{x})$, with $\Sigma(\tilde{x})$ solving the statedependent Riccati equation

$$
\Sigma(\tilde{x}) \tilde{A}(\tilde{x})+\tilde{A}^{T}(\tilde{x}) \Sigma(\tilde{x})-\Sigma(\tilde{x}) \tilde{B}(\tilde{x}) N^{-1} \tilde{B}^{T}(\tilde{x}) \Sigma(\tilde{x})+M=0
$$

or, rewritten in terms of the original variables, $L(x)=\Sigma(x) C^{T}(x) N^{-1}$ with $\Sigma(x)$ satisfying

$$
\Sigma(x) A^{T}(x)+A(x) \Sigma(x)-\Sigma(x) C^{T}(x) N^{-1} C(x) \Sigma(x)+M=0 .
$$


This fully state-dependent version of the Riccati equation can be very difficult to solve due to the $\Sigma(x) C^{T}(x) N^{-1} C(x) \Sigma(x)$ term, especially if the measurement function is complicated. To deal with this, we will make a simplification, linearizing the measurement for the purposes of finding $L(x)$. We wish to keep intact as much of the nonlinear nature of the problem as possible, though, so while we remove the state-dependence of $C(x)$, we keep $A(x)$ intact in the Riccati equation. Having done this, we can use the SDRE solution method described in Section 2 to find the state estimation gain

$$
L(x)=\Sigma(x) C_{0}^{T} N^{-1}
$$

from the state-dependent Riccati equation

$$
\Sigma(x) A^{T}(x)+A(x) \Sigma(x)-\Sigma(x) C_{0}^{T} N^{-1} C_{0} \Sigma(x)+M=0
$$

with $C_{0}=C(0)$. The nonlinear dynamics and measurement are also still intact in the main part of the estimator,

$$
\dot{x}_{e}=f\left(x_{e}\right)+B u\left(x_{e}, t\right)+L\left(x_{e}\right)\left(z-c\left(x_{e}\right)\right),
$$

as they are in the methods of Thau and others described above.

\section{Application to Test Problems}

\subsection{Simple Example System}

We test the tracking and state estimation methods in Sections 3 and 4 first on a simple example problem from [30], which was used as a test problem for the nonlinear control method survey in [1]. The nonlinear control system is given by

$$
\left[\begin{array}{c}
\dot{x}_{1} \\
\dot{x}_{2}
\end{array}\right]=\left[\begin{array}{cc}
0 & 1 \\
x_{1}^{2} & 0
\end{array}\right]\left[\begin{array}{l}
x_{1} \\
x_{2}
\end{array}\right]+\left[\begin{array}{l}
0 \\
1
\end{array}\right] u
$$

in a factorized form appropriate for the SDRE. The cost functional is given by

$$
J\left(x_{0}, u\right)=\frac{1}{2} \int_{0}^{\infty}\left(x^{T} Q x+u^{T} R u\right) d t
$$

or, slightly modified for the tracking problem,

$$
J\left(x_{0}, u\right)=\frac{1}{2} \int_{0}^{\infty}\left((y-r)^{T} Q(y-r)+u^{T} R u\right) d t .
$$

First we will consider just the feedback tracking control problem, with no state estimation involved. We will track the variable $y=x_{1}$, attempting to force it to jump up from 0 to 0.5 , hold, and then jump back to 0 (as shown in Figure 1). The weights in the cost function will be set to $Q=10$ and $R=1$, and the initial condition, of course, is at the origin. The numerical computation of the controls and solution of the systems are done with MATLAB code written by the authors, using the built-in functions "are" for solving constant-valued algebraic Riccati equations and "ode45" for solving ODE systems. The two-point boundary value problem for the tracking variable in the 
nonlinear case is solved with a final time of $T_{f}=15$, and 100 time discretization intervals. The first five terms of the SDRE power series solution are used.

The results of the tracking control problem are plotted in Figure 1 for the nonlinear tracking control described in Section 3 in equations (19)-(21), as well as a linear tracking control obtained by linearizing the problem and then using the standard linear techniques. The nonlinear control

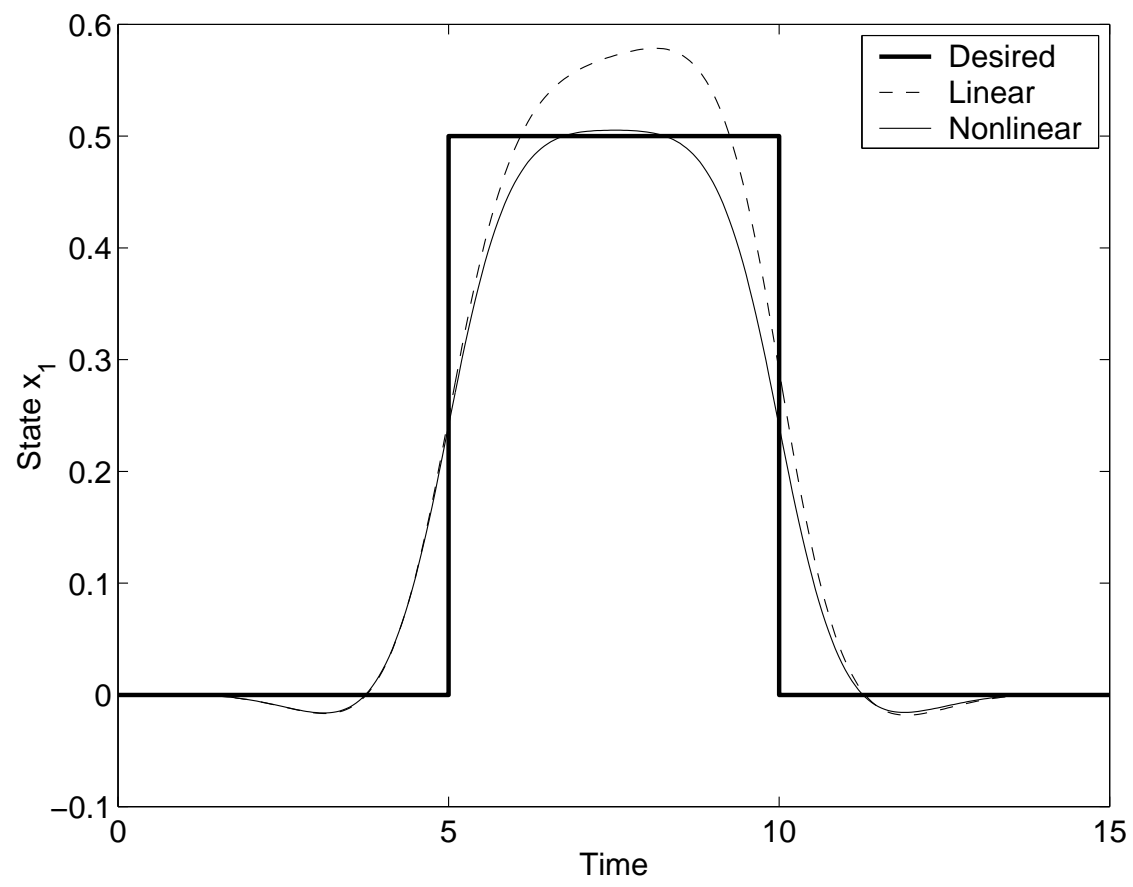

Figure 1: Comparison of feedback tracking controls on Example 1, with weight $Q=10$.

is obviously superior here, as the linear control drastically overshoots the maximum of the desired trajectory. Raising the cost function weight on the state to $Q=100$, as shown in Figure 2, brings the linearly controlled state down much closer to the nonlinear case, but the nonlinear control still produces better results.

Next, looking at a control problem with state estimation and without any tracking component, we will set the initial condition to $x_{0}=[1,0]^{T}$ and ask the control to force the system to 0 . The initial estimated state will be $\left(x_{e}\right)_{0}=[1.3,0]^{T}$, slightly off of the actual state, forcing the estimator to compensate. The estimation will be based on the nonlinear measurement $z=c(x)=x_{1}+x_{1} x_{2}$. In this problem we will set the cost function weights to $Q=10 I_{2}$ ( $I_{2}$ being a $2 \times 2$ identity matrix) and $R=1$, with the weights in the state estimator gain problem being $M=I_{2}$ and $N=1$. In Figure 3 we plot the actual and estimated states for two methods of state estimation described in Section 4. One is the method based on Thau's work, which is the nonlinear estimator in equation (26) using a linear gain found with a constant-valued Riccati equation (we will refer to this as the linear SE gain control). The other is the extension of this which is described in equations (27)-(29), a nonlinear estimator using a nonlinear gain from a state-dependent Riccati equation. The control using nonlinear SE gain performs slightly better than the linear SE gain control in this example, with the estimated state converging to the actual state faster and the state moving to 0 faster as 


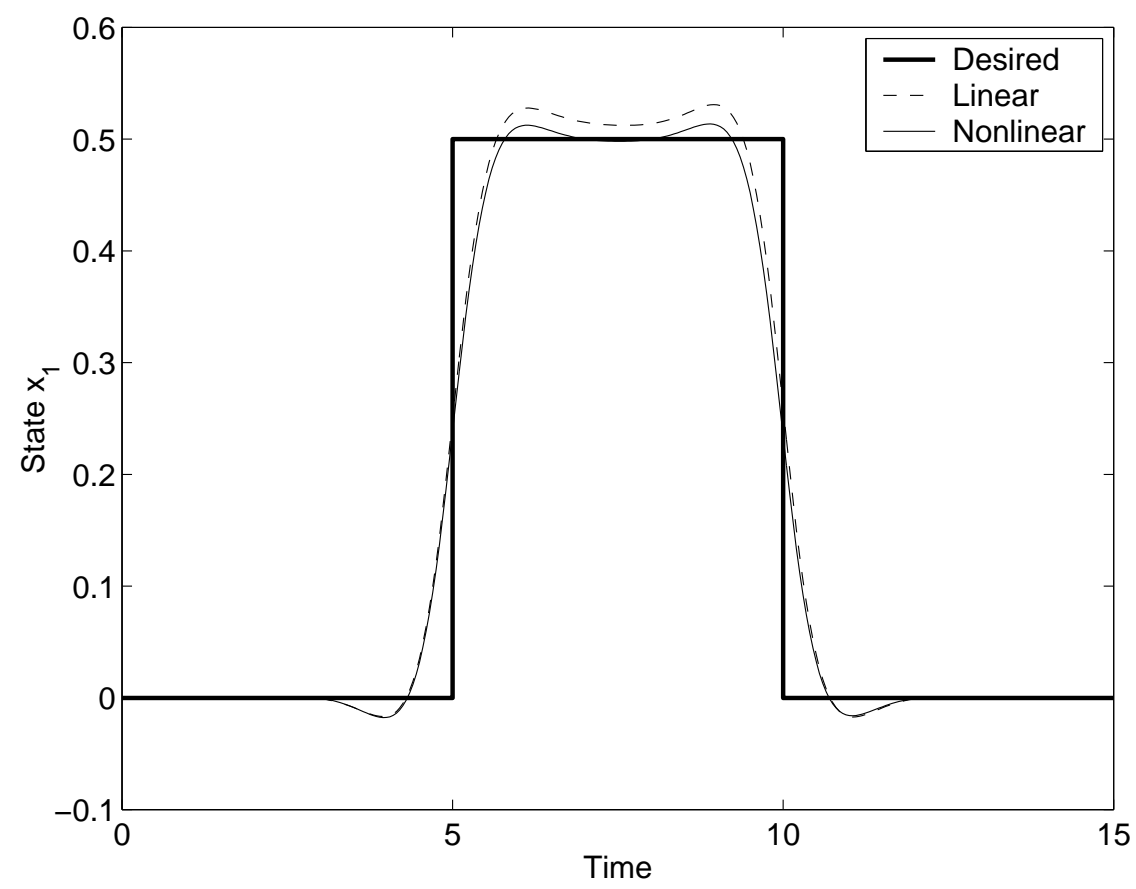

Figure 2: Comparison of feedback tracking controls on Example 1, with weight $Q=100$.

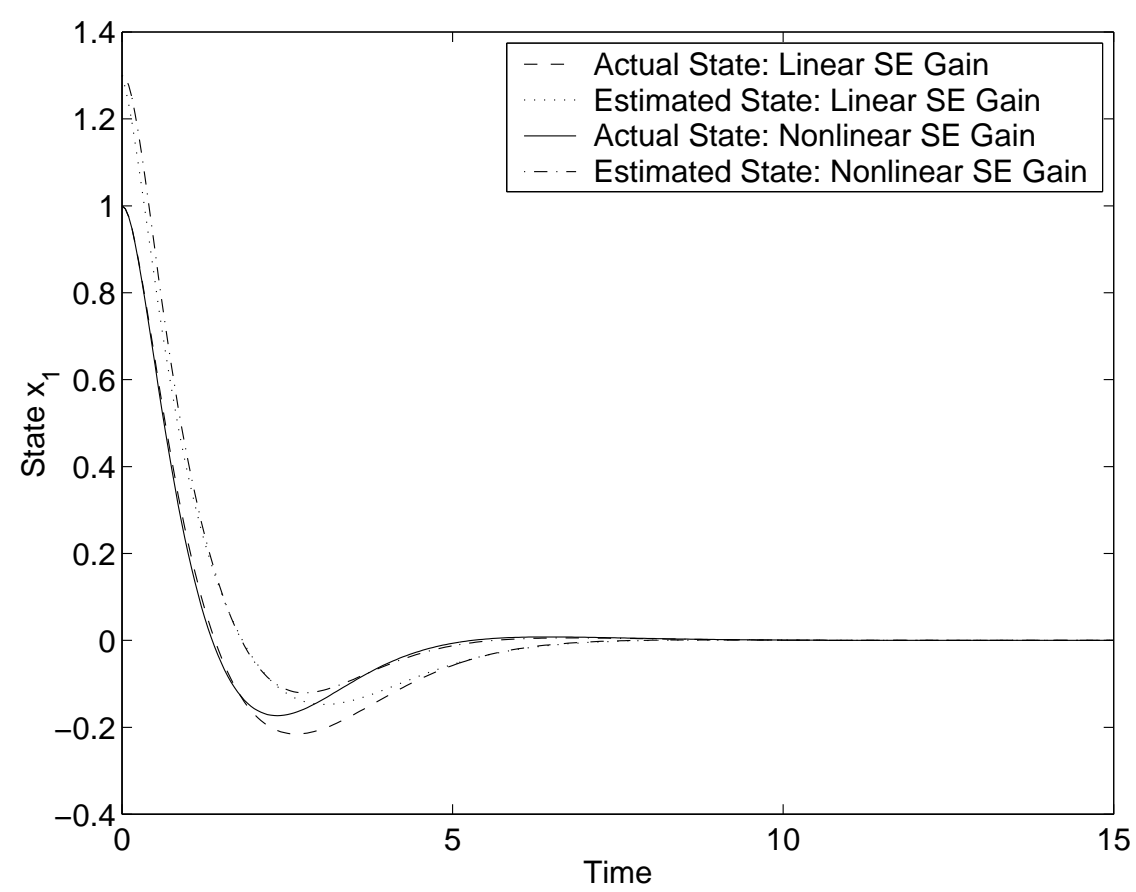

Figure 3: Actual and estimated states for feedback controls/state estimators in Example 1. 
well. A control found by completely linearizing the problem and finding a linear state estimator and control was tried on the problem but failed to yield state convergence.

State estimation is now added to the tracking control problem, using the same desired step function as in Figures 1 and 2. The weights in this case are $Q=10, R=1, M=10 I_{2}$ and $N=1$, and the measurement is $z=x_{1}+x_{1} x_{2}$ as before. The initial condition is $x_{0}=[0,0]^{T}$ and the initial estimated state is $\left(x_{e}\right)_{0}=[0.25,0]^{T}$. Figure 4 depicts the actual and estimated states $x_{1}$ which result from the feedback control combining the nonlinear tracking and state estimation techniques. The estimator converges very nicely to the actual state while tracking the desired trajectory well.

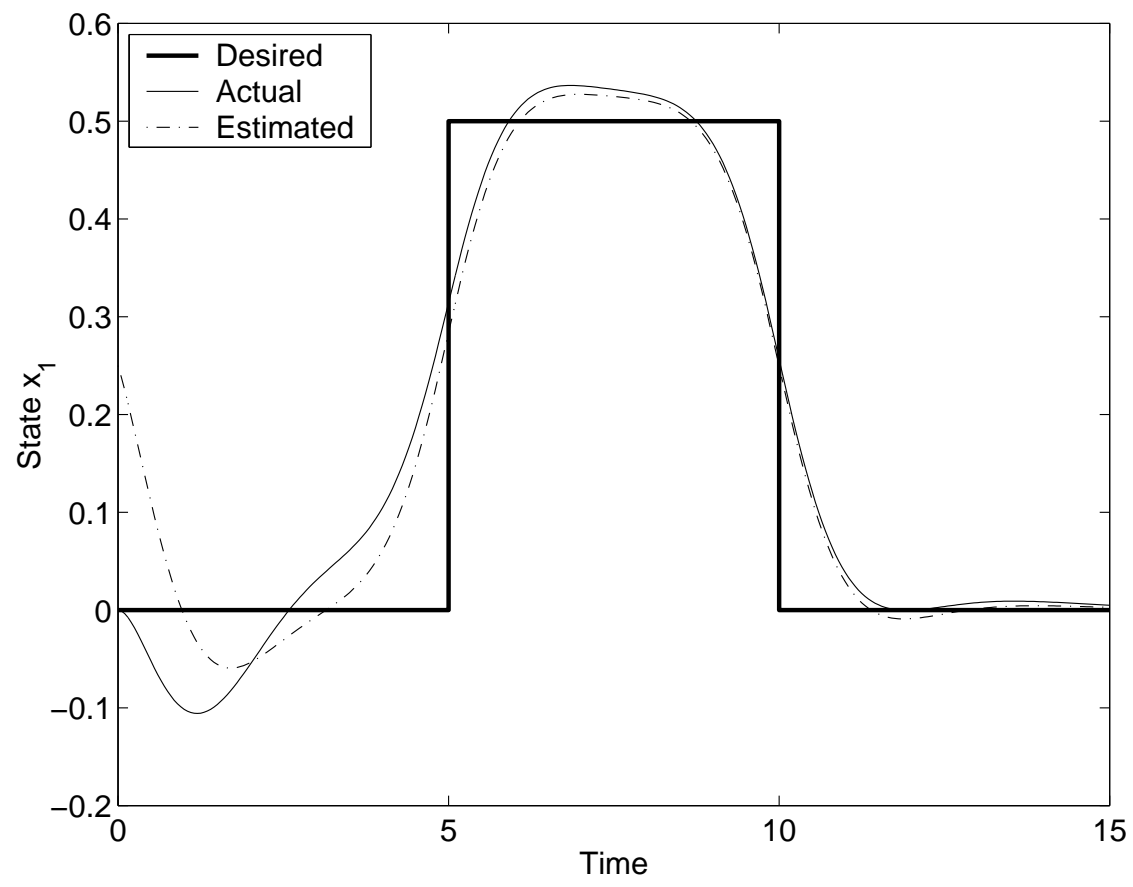

Figure 4: Actual and estimated states for nonlinear tracking control/state estimator in Example 1.

This fully nonlinear control is plotted, with a partially nonlinear control using the nonlinear tracking formula and the linear SE gain, and with the fully linear control found from the linearized system, in Figure 5. The fully nonlinear control performs better than the linear SE gain control in forcing the state to follow the desired trajectory (specifically in the latter half of the time period), while the fully linear control is far less effective.

Finally, we consider the same problem as in Figures 4 and 5, with both state estimation and tracking, except that instead of a deviation in the initial estimated state for which the estimator must compensate, there is instead added random noise in the problem. The noise consists of independent uniform distributions $\varepsilon_{1}(t)$ and $\varepsilon_{2}(t)$, with $\left|\varepsilon_{k}\right| \leq 0.1$ (20\% of the maximum desired $x_{1}$ trajectory), one added to the dynamics and the other to the measurement:

$$
\left\{\begin{aligned}
\dot{x} & =A(x) x+B u+\varepsilon_{1} \\
z & =x_{1}+x_{1} x_{2}+\varepsilon_{2}
\end{aligned}\right.
$$

In Figure 6 we compare the results of the fully nonlinear, linear SE gain, and fully linear controls 


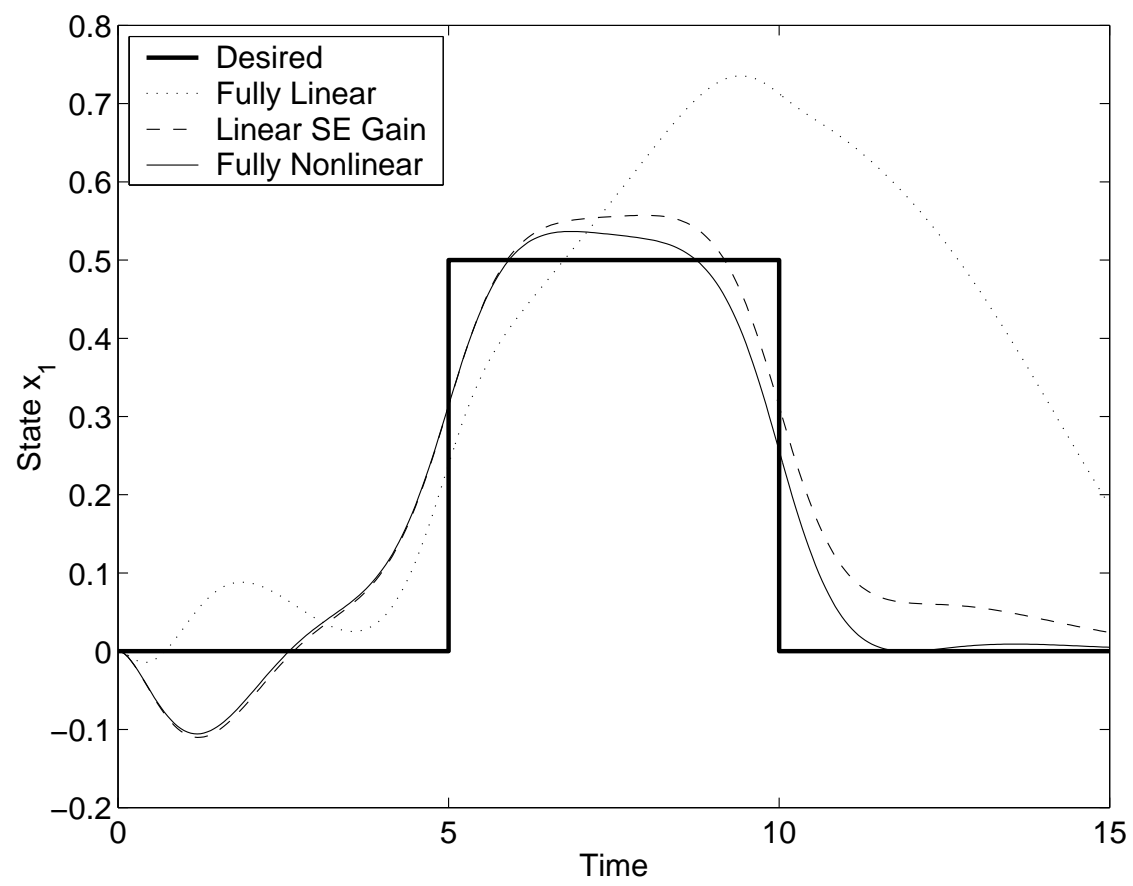

Figure 5: Comparison of tracking controls/state estimators on Example 1, with inaccurate $\left(x_{e}\right)_{0}$.

applied to this problem. The fully nonlinear and the linear SE gain controls yield very similar results here, and, as in Figure 5, both perform much better than the fully linear control.

\subsection{Flight Dynamics Simulation Example}

The second example to which we apply the state estimation and tracking control methods discussed in Sections 3 and 4 is a modified version of the flight dynamics example from [31] (this was also used as test problem in [1]). The system is given by

$$
\dot{x}=\left(A_{0}+x_{2} A_{N L}\right) x+B u,
$$

where the matrices $A_{0}, A_{N L}$ and $B$ are given by:

$$
\begin{aligned}
A_{0}= & {\left[\begin{array}{ccccc}
-0.0443 & 1.1280 & 0.0 & -0.0981 & 0.0 \\
-0.0490 & -2.5390 & 1.0 & 0.0 & -0.0854 \\
-0.0730 & 19.3200 & -2.2700 & 0.0 & 22.6834 \\
0.0490 & 2.5390 & 0.0 & 0.0 & 0.0854 \\
0.0 & 0.0 & 0.0 & 0.0 & 20.0
\end{array}\right] } \\
A_{N L}= & {\left[\begin{array}{ccccc}
-0.2317 & 0.0 & 0.0 & 0.0 & 0.0 \\
-1.2760 & -0.7922 & 0.0 & 0.0 & 0.0206 \\
0.1020 & 64.2940 & -13.9710 & 0.0 & -5.4167 \\
1.2760 & 0.7922 & 0.0 & 0.0 & -0.0206 \\
0.0 & 0.0 & 0.0 & 0.0 & 0.0
\end{array}\right] }
\end{aligned}
$$




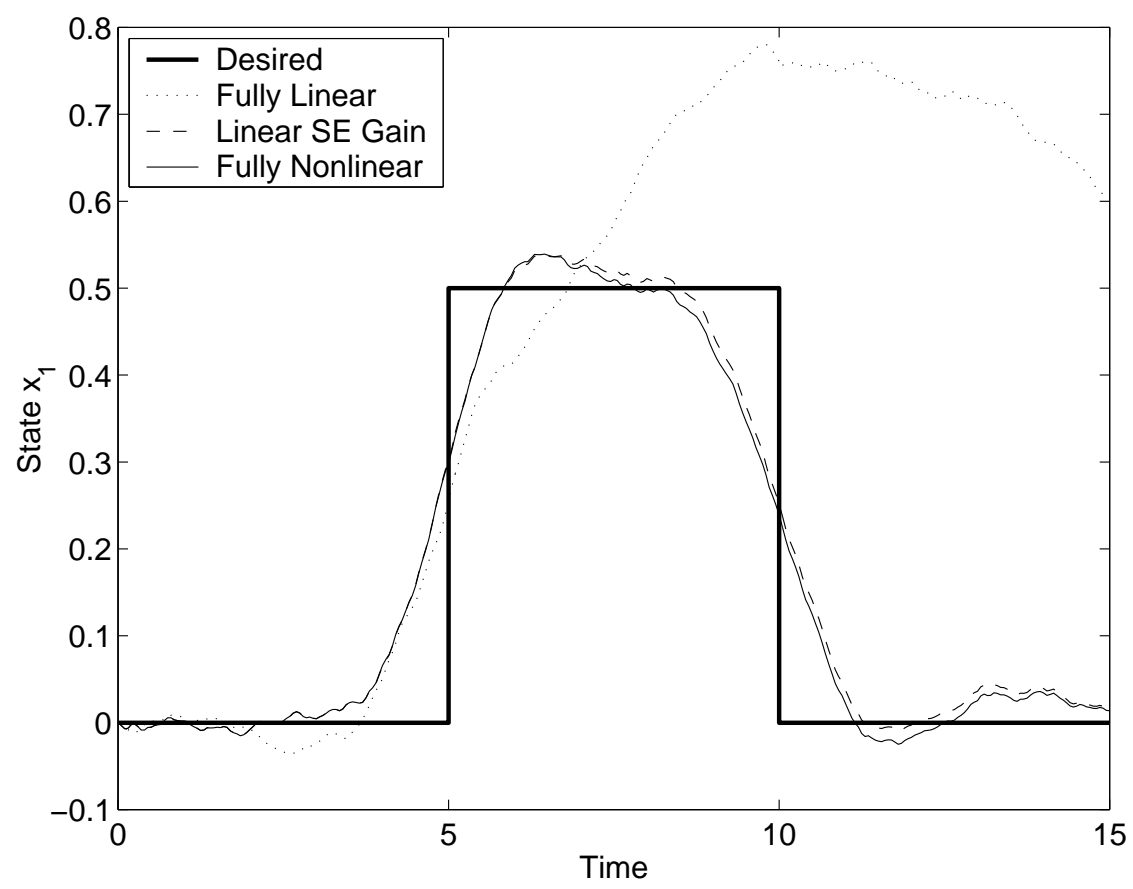

Figure 6: Comparison of tracking controls/state estimators on Example 1, with noise.

$$
B=\left[\begin{array}{lllll}
0.0 & 0.0 & 0.0 & 0.0 & 20.0
\end{array}\right]^{T} .
$$

The cost functional to be minimized is

$$
J\left(x_{0}, u\right)=\frac{1}{2} \int_{0}^{\infty}\left((y-r)^{T} Q(y-r)+u^{T} R u\right) d t .
$$

when in tracking problem form. The state variables in this model represent the flight conditions of the aircraft: $x_{1}$ is the deviation of the velocity from the level flight trim value of $1(100 \mathrm{~m} / \mathrm{s}$ ) (given in units of $(100 \mathrm{~m} / \mathrm{s})), x_{2}$ is the deviation of the angle of attack from the trim value of $4.2(\pi / 180)$ radians, $x_{3}$ is the pitch rate in $\mathrm{rad} / \mathrm{s}, x_{4}$ is the flight path angle in radians, and $x_{5}$ is the deviation of the canard deflection angle in radians from the trim value, which is not given. The control $u$ is the input canard deflection in radians. The canards are control flaps which can deflect downward by up to $90(\pi / 180)$ radians. (The meaning of the model is described in more detail in [31].)

In the first test we consider a control problem with state estimation but no tracking. The feedback control will attempt to force the state variables to 0 from an initially large angle of attack, given by an initial condition of $x_{0}=(0,15(\pi / 180), 0,0,0)^{T}$. However, the initial estimated state is $\left(x_{e}\right)_{0}=(0,20(\pi / 180), 0,0,0)^{T}$. The measurement for the estimator is of the velocity and the canard deflection, so that $z=c(x)=\left[x_{1}, x_{5}\right]^{T}$. The cost functional weights are given by $Q=I_{5}$ and $R=100$, and the weights for the estimator gain problem are $M=100 I_{5}$ and $N=I_{2}$. Figure 7 depicts the actual and estimated states for this problem using the linear SE gain (Thau) control and the nonlinear SE gain control found with an SDRE solution. For each method it takes some time for the estimated state to converge to the actual state, and for both to be forced to 0 , but they both do so in a smooth manner. There is a larger oscillation noticeable in the linear SE gain control, 


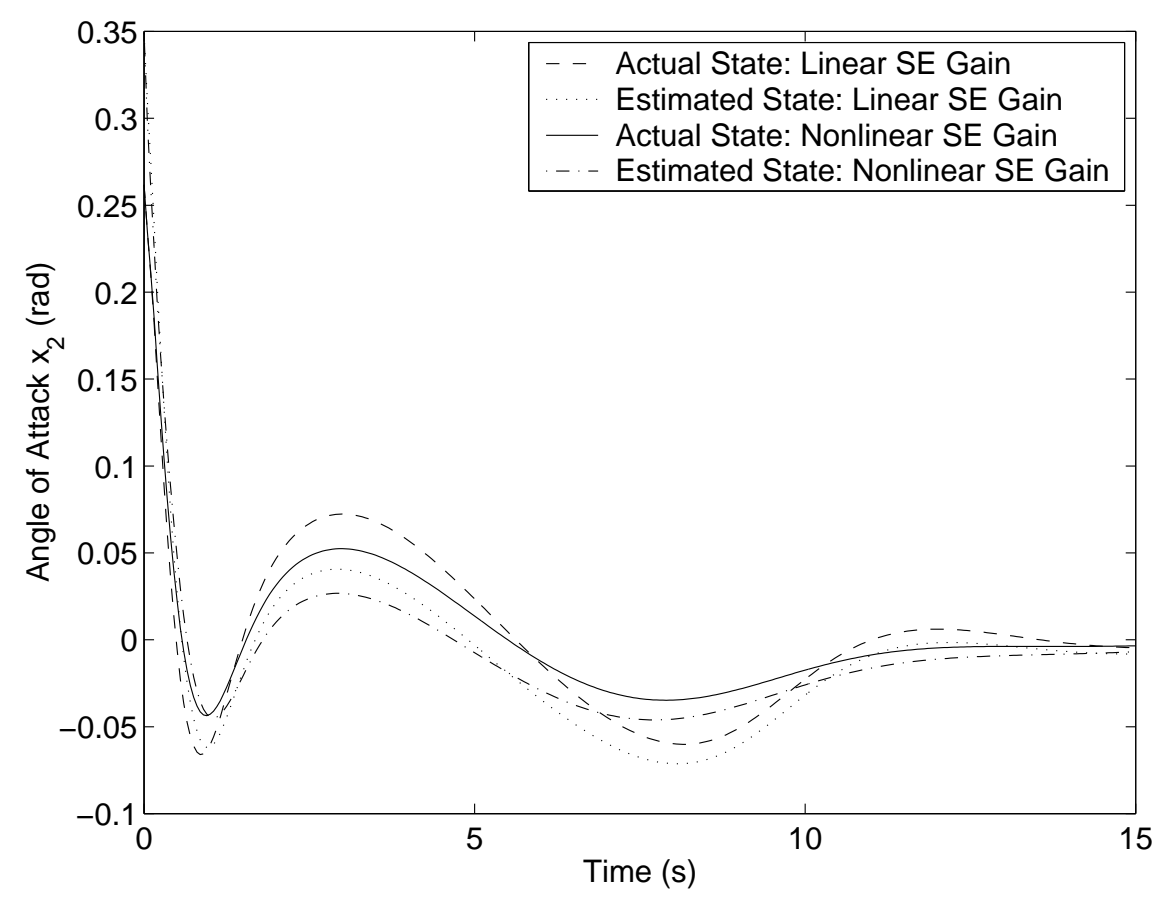

Figure 7: Actual and estimated states for feedback controls/state estimators in Example 2.

causing slower convergence. On the other hand, with the fully linear state estimation and control algorithm applied, the system remains unstable and diverges to infinity.

Next we will consider a problem with tracking but no state estimation. The objective is to track a desired flight path angle $x_{4}$, increasing it gradually from 0 up to $45(\pi / 180)$, holding, and then returning to 0 (as shown in Figure 8). The initial condition is therefore at the origin, and the cost weights are $Q=1$ and $R=10$. The results are plotted in Figure 8 for the nonlinear and linear tracking controls. Here the nonlinear control does not perform substantially better than the linear control; in fact the linear control yields better results at the top of the ramp up to $45(\pi / 180)$ ) though in the late time period the linear control returns to 0 more slowly than the nonlinear.

In considering a problem with both tracking and state estimation, we use the same desired trajectory as before, forcing the flight path angle $x_{4}$ from 0 up to $45(\pi / 180)$ and back. Estimation is added using a measurement of the velocity, angle of attack, and canard deflection: $z=\left[x_{1}, x_{2}, x_{5}\right]^{T}$. The weights are $Q=1, R=1, M=100 I_{5}$ and $N=I_{3}$. The actual state starts at the origin, as in the previous tracking problem, but the estimated state starts slightly off the actual, at $\left(x_{e}\right)_{0}=$ $(0,0,0,5(\pi / 180), 0)^{T}$. Figure 9 depicts the estimated state almost converging to the actual state by the time of the desired $x_{4}$ increase, and remaining close to the actual state for the rest of the time period. In Figure 10 we plot the actual state when controlled using our fully nonlinear algorithm, as well as when using the linear SE gain control, and the fully linear control. It can be seen that the linear control overshoots significantly at the top of the ascent and is very slow to return to 0 . The other two methods produce virtually identical results, the difference indiscernable in the plots in Figure 10.

Finally, we alter the problem used in Figures 9 and 10, involving both state estimation and 


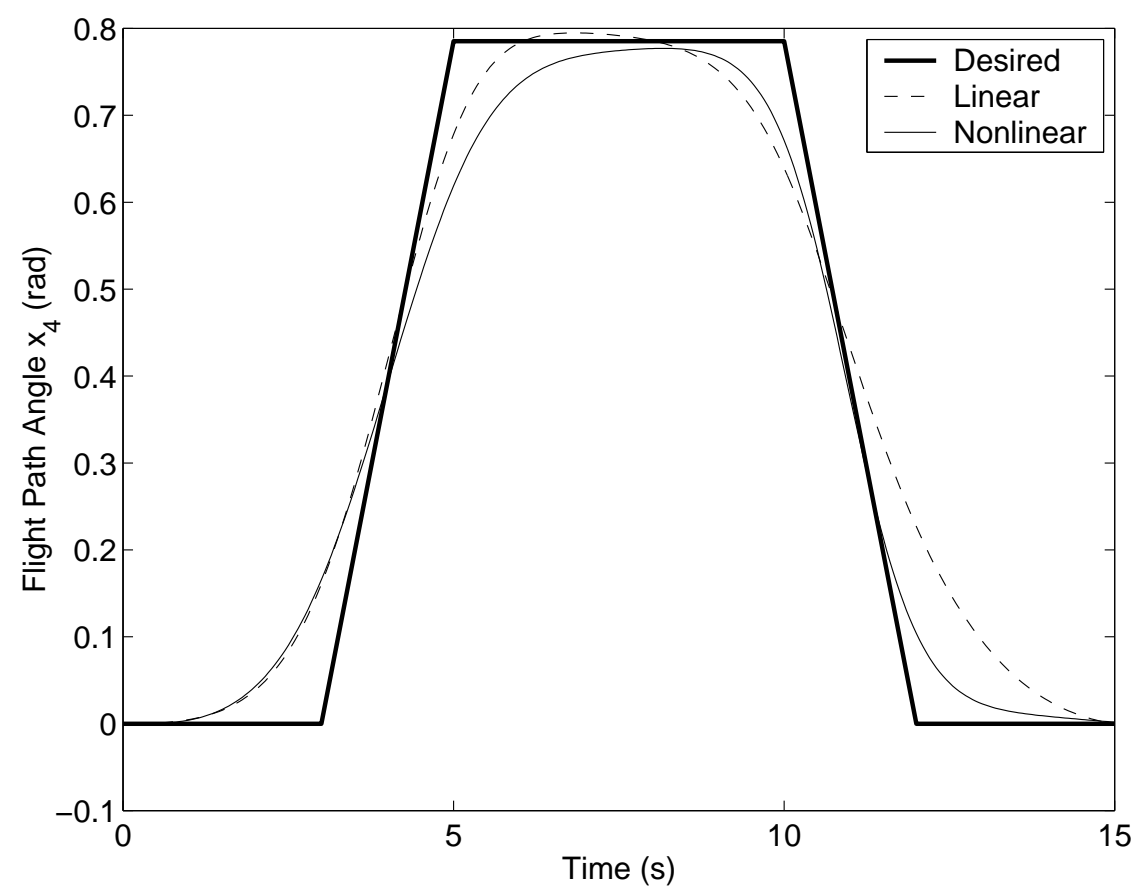

Figure 8: Comparison of feedback tracking controls on Example 2.

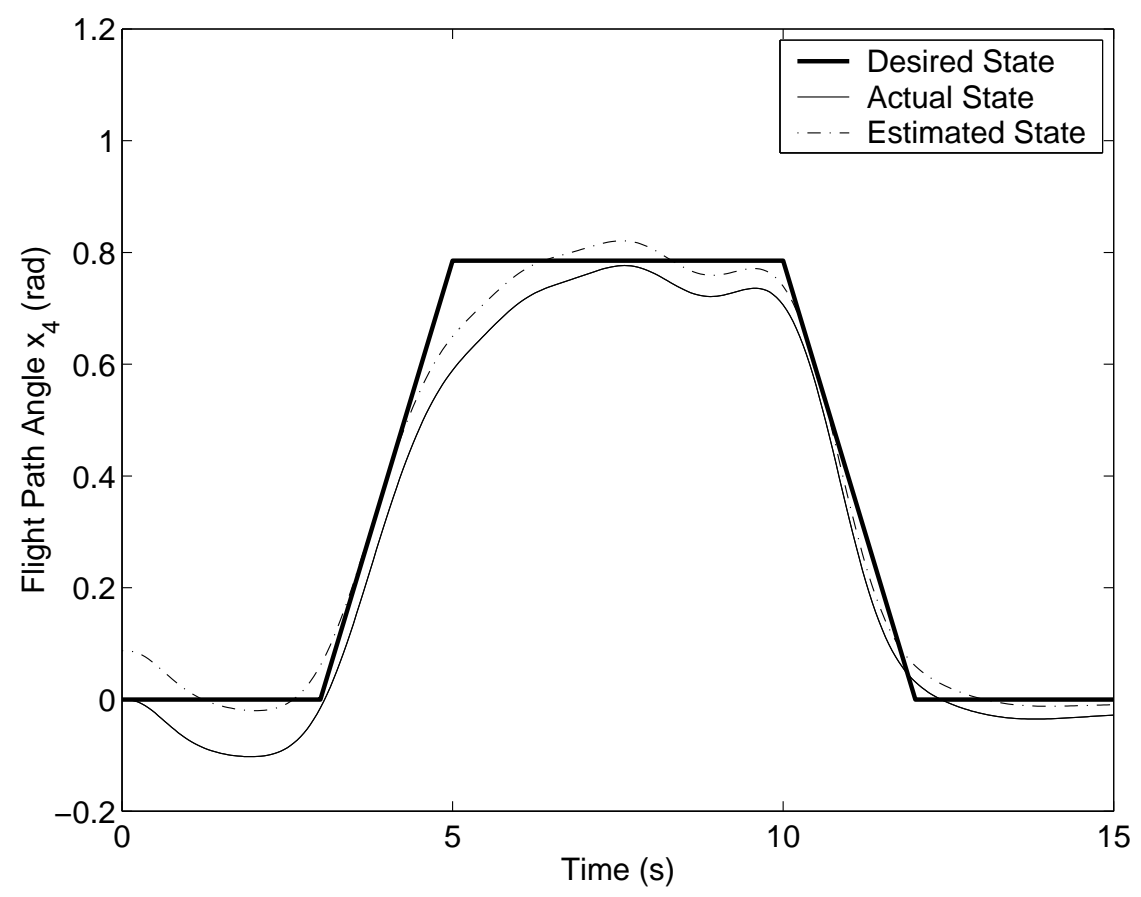

Figure 9: Actual and estimated states for nonlinear tracking control/state estimator in Example 2. 


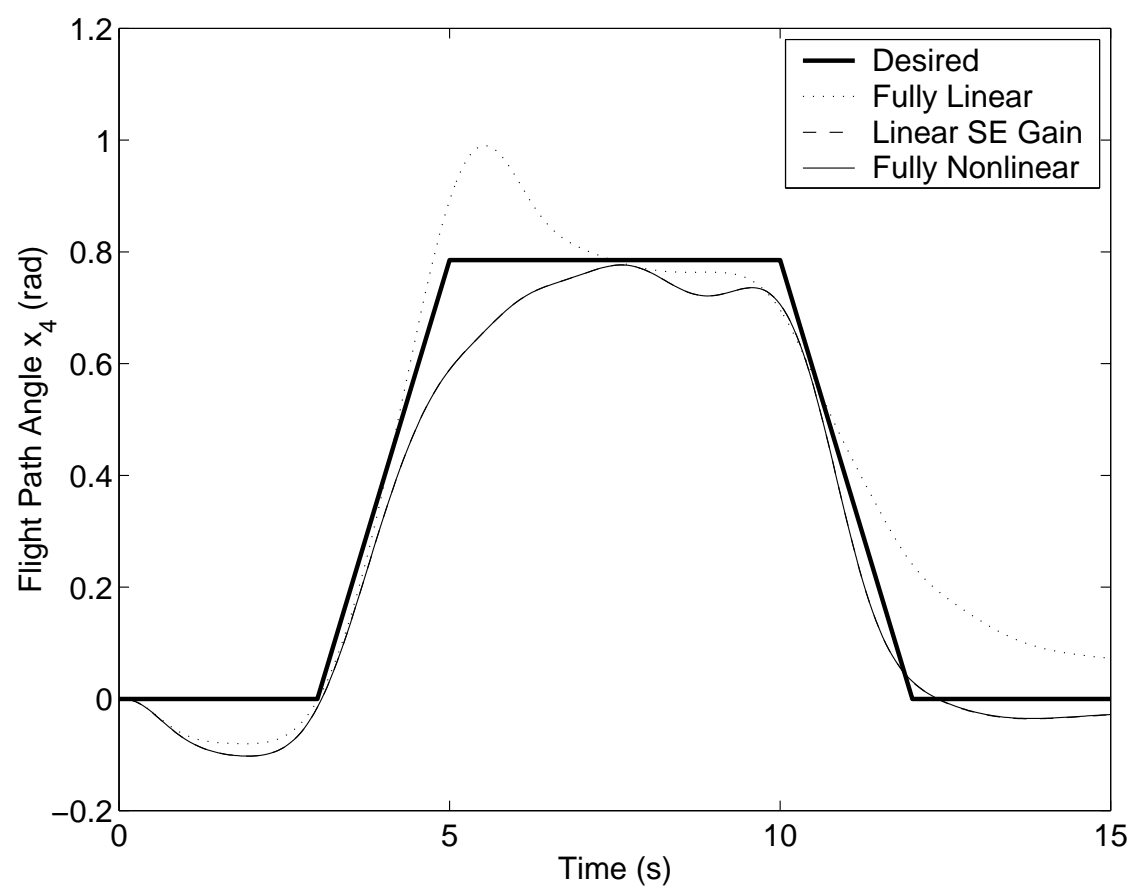

Figure 10: Comparison of tracking controls/state estimators on Example 2, with inaccurate $\left(x_{e}\right)_{0}$.

tracking, by removing the deviation in the initial estimated state and adding random noise to the problem. The independent uniform distributions $\varepsilon_{1}(t)$ and $\varepsilon_{2}(t)$, with $\left|\varepsilon_{k}\right| \leq 2.25(\pi / 180)(5 \%$ of the maximum desired flight path angle, and an even larger percentage of the actual state variables at most times), are added to the dynamics and the measurement respectively:

$$
\left\{\begin{aligned}
\dot{x} & =\left(A_{0}+x_{2} A_{N L}\right) x+B u+\varepsilon_{1} \\
z & =\left[x_{1}, x_{2}, x_{5}\right]^{T}+\varepsilon_{2} .
\end{aligned}\right.
$$

The results of the fully nonlinear, linear SE gain, and fully linear controls applied to this problem are plotted in Figure 11, where it can be seen that the two nonlinear controls are again very similar, and that both perform better than the linear control at tracking the desired trajectory both at its maximum and as it returns to 0 .

\section{Conclusions}

In this paper we have considered the method for feedback control of nonlinear systems using the state-dependent Riccati equation and extended it into a feedback tracking control method. We have also modified the state estimation method for nonlinear systems established in the literature to include a nonlinear gain function found through a state-dependent Riccati equation. Application of these new techniques to two selected example problems provided significant control authority and distinct advantages in comparison with the linear methods.

As mentioned in earlier sections, there are some drawbacks and restrictions to the new techniques which must be considered. The power series solution of the SDRE method grows inaccurate when the 


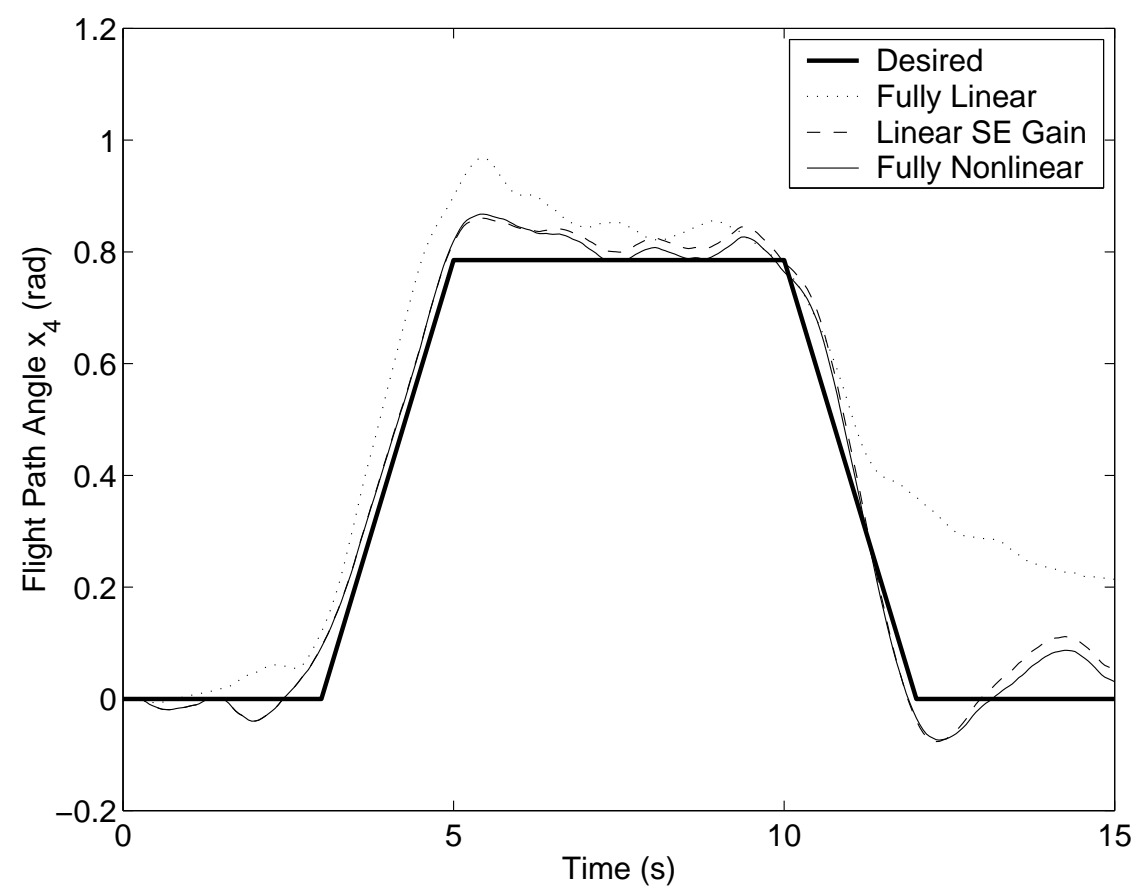

Figure 11: Comparison of tracking controls/state estimators on Example 2, with noise.

states move farther from the origin, something which is of particular concern in a tracking problem. In solving the tracking variable equation by coupling it with a nominal state equation, one tacitly assumes a good prediction of the actual state behavior for the control to be effective. There are limitations on the types of problems to which the SDRE approach can be applied and on the types of signals which can be tracked, and the SDRE for obtaining the nonlinear state estimation gain uses only a linearized version of the measurement function. While these are nontrivial factors to consider, the methods described here for tracking control and state estimation are still applicable to a large class of important control problems, and their performance on the chosen examples provides improvement (in places dramatic improvement) when compared to previously established control techniques. 


\section{References}

[1] Beeler, S. C., Tran, H. T., and Banks, H. T., "Feedback Control Methodologies for Nonlinear Systems," Journal of Optimization Theory and Applications, to appear.

[2] Anderson, B. D. O., and Moore, J. B., Optimal Control: Linear Quadratic Methods, Englewood Cliffs, New Jersey: Prentice-Hall, 1990.

[3] Lewis, F. L., and Syrmos, V. L., Optimal Control, New York: Wiley, 1995.

[4] Garrard, W. L., "Suboptimal Feedback Control for Nonlinear Systems," Automatica, Vol 8, pp. 219-221 (1972).

[5] Pearson, J. D., "Approximation Methods in Optimal Control," Journal of Electronics and Control, Vol 13, pp. 453-465 (1962).

[6] Burghart, J. A., "A Technique for Suboptimal Control of Nonlinear Systems," IEEE Transactions on Automatic Control, Vol 14, pp. 530-533 (1969).

[7] Wernli, A. and Cook, G., "Suboptimal Control for the Nonlinear Quadratic Regulator Problem," Automatica, Vol 11, pp. 75-84 (1975).

[8] Krikelis, N. J. and Kiriakidis, K. I., "Optimal Feedback Control of Non-linear Systems," International Journal of Systems Science, Vol 23, pp. 2141-2153 (1992).

[9] Cloutier, J. R., D'Souza, C. N., and Mracek, C. P., "Nonlinear Regulation and Nonlinear $H_{\infty}$ Control Via the State-Dependent Riccati Equation Technique: Part 1. Theory," Proceedings of the First International Conference on Nonlinear Problems in Aviation and Aerospace, Daytona Beach, FL, May 1996.

[10] Thau, F. E., "Observing the State of Non-linear Dynamic Systems," International Journal of Control, Vol 17, pp. 471-479 (1973).

[11] Kou, S. R., Elliott, D. L., and Tarn, T. J., "Exponential Observers for Nonlinear Dynamic Systems," Information and Control, Vol 29, pp. 204-216 (1975).

[12] Mielczarski, W., "Observing the State of a Synchronous Generator - Part 1. Theory," International Journal of Control, Vol 45, pp. 987-1000 (1987).

[13] Hu, X., "On State Observers for Nonlinear Systems," Systems and Control Letters, Vol 17, pp. 465-473 (1991).

[14] Krener, A. J., and Isidori, A., "Linearization by Output Injection and Nonlinear Observers," Systems and Control Letters, Vol 3, pp. 47-52 (1983).

[15] Bestle, D., and Zeitz, M., "Canonical Form Observer Design for Non-linear Time-Variable Systems," International Journal of Control, Vol 38, pp. 419-431 (1983). 
[16] Krener, A. J., and Respondek, W., "Nonlinear Observers with Linearizable Error Dynamics," SIAM Journal of Control and Optimization, Vol 23, pp. 197-216 (1985).

[17] Zeitz, M., "The Extended Luenberger Observer for Nonlinear Systems," Systems and Control Letters, Vol 9, pp. 149-156 (1987).

[18] Xia, X., and Gao, W., "Nonlinear Observer Design by Observer Error Linearization," SIAM Journal of Control and Optimization, Vol 27, pp. 199-216 (1989).

[19] Gauthier, J. P., Hammouri, H., and Othman, S., "A Simple Observer for Nonlinear Systems Applications to Bioreactors," IEEE Transactions on Automatic Control, Vol 37, pp. 875-880 (1992).

[20] Soroush, M., "Nonlinear State-Observer Design with Application to Reactors," Chemical Engineering Science, Vol 52, pp. 387-404 (1997).

[21] Ciccarella, G., Dalla Mora, M., and Germani, A., "A Luenberger-Like Observer for Nonlinear Systems," International Journal of Control, Vol 57, pp. 537-556 (1993).

[22] Valluri, S., and Soroush, M., "Nonlinear State Estimation in the Presence of Multiple Steady States," Industrial and Engineering Chemistry Research, Vol 35, pp. 2645-2659 (1996).

[23] Baumann, W. T., and Rugh, W. J., "Feedback Control of Nonlinear Systems by Extended Linearization," IEEE Transactions on Automatic Control, Vol 31, pp. 40-46 (1986).

[24] Walcott, B. L., and Zak, S. H., "Observation of Dynamical Systems in the Presence of Bounded Nonlinearities/Uncertainties," Proceedings of the Twenty-fifth IEEE Conference on Decision and Control, Athens, Greece, pp. 961-966 (1986).

[25] Tsinias, J., "Further Results on the Observer Design Problem," Systems and Control Letters, Vol 14, pp. 411-418 (1990).

[26] Walcott, B. L., Corless, M. J., and Zak, S. H., "Comparative Study of Non-linear StateObservation Techniques," International Journal of Control, Vol 45, pp. 2109-2132 (1987).

[27] Banks, H. T., Smith, R. C., and Wang, Y., Smart Material Structures: Modeling, Estimation and Control, Chichester, England: Wiley, 1996.

[28] Lasiecka, I. and Triggiani, R., Differential and Algebraic Riccati Equations with Application to Boundary/Point Control Problems, New York: Springer-Verlag, 1991.

[29] Hammett, K. D., Hall, C. D., and Ridgely, D. B., "Controllability Issues in Nonlinear StateDependent Riccati Equation Control," Journal of Guidance, Control and Dynamics, Vol 21, pp. 767-773 (1998).

[30] Markman, J. and Katz, I. N., "An Iterative Algorithm for Solving Hamilton Jacobi Type Equations," preprint (1999).

[31] Garrard, W. L., Enns, D. F., and Snell, S. A., "Nonlinear Feedback Control of Highly Manoeuvrable Aircraft," International Journal of Control, Vol 56, pp. 799-812 (1992). 